\title{
Gute Berufsaussichten im Gartenbau
}

\section{Erste blühende Unternehmensmesse an der Hochschule Osnabrück zieht positive Bilanz}

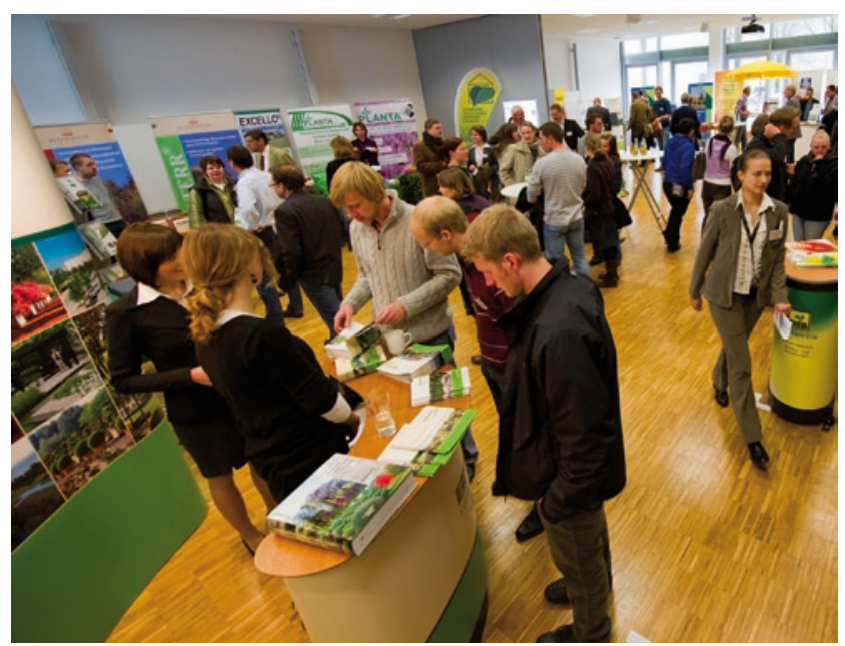

Firmen aus der gesamten Gartenbaubranche präsentierten sich den Studierenden der Hochschule Osnabrück

Unternehmen aus dem gesamten Bundesgebiet waren erstmalig zu Gast an der Fakultät Agrarwissenschaften und Landschaftsarchitektur der Hochschule Osnabrück und präsentierten den Studierenden vorwiegend die Substratund Düngemittelmittelbranche des Gartenbaus. Aber auch Firmen aus dem Produktionsgartenbau, gartenbaulichen Handel sowie Forschungs- und Entwicklungseinrichtungen waren auf der Messe vertreten und verdeutlichten die ganze Bandbreite an Einstiegsmöglichkeiten, die sich Absolventen nach ihrem Studium bieten.
Die Unternehmensvertreter beantworteten den Studierenden Fragen rund um die Berufsaussichten im Gartenbau. Welche Arbeitgeber gibt es überhaupt auf dem Markt und welche Anforderungen stellen sie an ihre Mitarbeiter? Die Studierenden machten rege Gebrauch von der Möglichkeit, ihre Arbeitgeber von morgen kennen zu lernen. Aber auch für Absolventen bot sich eine gute Gelegenheit, Unternehmenskontakte zu knüpfen. So entdeckten einige ehemalige Studierende, die sich gerade in der Bewerbungsphase befinden, ihnen bislang noch unbekannte Arbeitgeber auf der Messe.

Die Organisatorinnen der Messe, Marina Abramovskij und Sabrina Hoffmann vom Career Center der Hochschule, sind mit dem Ergebnis ihrer Arbeit zufrieden: „Die Resonanz von Studierenden und Unternehmensvertretern war durchweg positiv - beide Seiten berichteten von guten Gesprächen, die im besten Fall zu der ein oder anderen Einstellung führen werden“. Ein weiterer positiver Nebeneffekt - die Unternehmensvertreter nutzten auch untereinander die Gelegenheit sich miteinander bekannt zu machen, um neue Netzwerke zu knüpfen.

Die erste blühende Unternehmensmesse Gartenbau fand im Rahmen der Kontaktstudientage der Fakultät Agrarwissenschaften und Landschaftsarchitektur statt, die dieses Jahr mit 250 Teilnehmern aus der gesamten grünen Branche besonders erfolgreich war.

M. Abramovskij $(\bowtie)$

Career Center der FH Osnabrück, Standort Haste

E-Mail: m.abramovskij@fh-osnabrueck.de 